\title{
EDITORIAL
}

\section{Preparing for the next Influenza Pandemic: Bangladesh Perspective}

The threat of an influenza pandemic looms large over the world. Many experts believe that another influenza pandemic is virtually impossible to avoid. Within one hundred years the world has witnessed three major influenza pandemics: the 1918-19 "Spanish flu", the 1957-58 "Asian flu," and the 196869 pandemic or "Hong Kong flu". The most devastating of the three, the 1918-19 "Spanish flu" was responsible for an estimated 40 million death worldwide. The currently circulating H5N1 subtype of Influenza A virus is considered as the most likely cause of the next influenza Pandemic. The spread of the virus to Europe and Africa and continued human infections in Southeast Asia have further heightened pandemic concern. The world still remains at Phase 3 of the Pandemic Alert Period as declared by WHO since January 2004. That indicates the novel influenza virus subtype (H5N1) is causing sporadic human cases, but yet develop capacity to transmit efficiently among humans. Highly pathogenic Avian Influenza (H5N1) among poultry was first reported in Bangladesh in later half of March 2007. Since then $\mathrm{HPAI} / \mathrm{H} 5 \mathrm{~N} 1$ has been reported in 12 districts both in organized and backyard poultry including homereared pigeons in some affected areas. To mitigate the spread of HPAI/H5N1, department of Livestock culled more than 100000 poultry in the affected regions. So far no human case has been reported in Bangladesh. The H5N1 subtype of Influenza A virus is novel to human and able to cause serious disease in human with a high case fatality rate approaching $60 \%$, but it has yet to acquire the ability to transmit efficiently among the human. Most of the human infections to date have resulted from direct contact with infected poultry, particularly back yard poultry. There are several reports of human to human transmission of H5N1, particularly among close household contacts, but evidence gathered so far indicated that it has not yet acquired the capability to maintain a sustained chain of transmission among humans. Once this particular virus acquires the ability to maintain a sustained human to human transmission, pandemic will ensue. Therefore preparedness for a possible influenza pandemic caused by Highly Pathogenic Avian Influenza A subtype H5N1 (HPAI/H5N1) has become a global priority.

Preparedness for the pandemic influenza in Bangladesh has commenced from mid 2005. In 2006, the "National Avian Influenza and Human Pandemic Influenza Preparedness and Response Plan Bangladesh 2006-2008" was approved by the government. As per proposed plan in during the phase where only animal infection of H5N1 is reported, ministry of fisheries and livestock will lead the pandemic preparedness campaign, but once a single human case become notified, ministry of health and family welfare will take the lead. However during pandemic the highest executive of the government will take the help of the program. To supervise and implementation of the plan several committees has been formed from national to upazila level both in the ministry of livestock and in the ministry of health and family welfare with multisectoral committees for coordination at all levels. All divisional, district and upazila level health managers of the country has been oriented on avian/human pandemic influenza through series of workshops started from early 2006. The Ministry of Health and Family Welfare (MOHFW) through Directorate General of Health Services (DGHS) has already stockpiled anti-viral drug Oseltamivir and other supportive medication for management and prophylaxis of avian influenza $\mathrm{H} 5 \mathrm{~N} 1$. As delineated in the plan, isolation units in district hospitals with ventilators for respiratory support are currently in the process of development. WHO has already procured and in the process of further procurement of adequate number of Personal Protective Equipments (PPE) for rapid response teams and in case of suspected outbreaks. Anti-viral 
vaccines for seasonal influenza are also being stockpiled for health care workers and rapid responders to decrease the possibility of mixing of avian and human influenza viruses. Institute of Epidemiology, Disease Control and Research (IEDCR), which is the nationally mandated organization for outbreak investigation, has been identified as the focal institute for pandemic preparedness activities. To this end Initiatives have been taken to set up a modern Influenza referral laboratory with skilled laboratory personnel at IEDCR. Soon the nationwide surveillance of Influenza Like Illness (ILI) will begin in 18 designated district hospitals as sentinel sites and over time it will phase-wise cover all 64 districts Hospitals for surveillance of Viral Pneumonia.

The national plan has also proposed an eleven member core District Rapid Response Team (DRRT) headed by the civil surgeons in each districts. The primary responsibility of the DRRT is to respond to any outbreak of suspected Avian Influenza Infection in human and supports the initial 72 hours of investigation. IEDCR has already completed intensive module based training of all 64 DRRTs on rapid response, infection control and risk communication on Avian/Human Pandemic Influenza. District based training of the Upazila Rapid Response Team (URRT) will soon be started. This training will also reach least 50 health care workers and volunteers from each union, who will take part in active dissemination of awareness messages to the community, that are already been developed by Technical Working Group on risk communication.

Although we know that the widespread use of a pandemic vaccine should be the central strategy for protection of human health during a pandemic event but the vaccine will not be available during the initial peak period of a pandemic. Even then more time will be elapsed until the vaccine become available to the resource poor countries. The other option we are left with is the use Oseltamivir for treatment and prophylaxis.. Therefore recognition of the cluster of cases and early initiation of management is of pivotal importance. Oseltamivir resistance $\mathrm{H} 5 \mathrm{~N} 1$ cases has been identified in Viet Nam and many experts apprehend that possibility of rate of resistance to
Oseltamivir will also be very high during pandemic scenario. Therefore it is imperative that the use of this drug must be rigorously controlled. Currently the drug is available only thorough Government health system and to be used in accordance to the approved guidelines. It should remain as such because indiscriminate use of this drug will result in more resistance through a selection pressure on the circulating influenza viruses. In absence of vaccine this drug is crucial for pandemic containment.

It is also vital to prepare contingency plan clearly outlining strategies for strengthening health system response capacity in clinical care and isolation practices in light of perceived enhanced demand. We need to ensure medical readiness by ensuring those health care workers, including physicians, nurses, and all allied health personnel are able to perform their duties during an influenza pandemic. By its very nature, their work puts these individuals at higher risk during a pandemic. To this end, the Government needs to ensure the availability of influenza vaccinations, antiviral treatment and prophylaxis, a possible risk allowance plan to eliminate barriers to health care workers' participation during an influenza pandemic. There is also strong need to act now to establish a national strategy to ensure a coordinated continuum of care during a pandemic. This involves building national, regional, and local health care systems capable of responding to a mass casualty event by establishing the protocols necessary to develop and sustain medical surge capacity though providing training, education of the medical personnel and developing a detailed, evidence-based guidelines. It is also imperative to institute and strengthen safe respiratory hygiene practices and promote adoption of standard and transmission based infection control precaution practices in health care settings as well as at the individual and community level. National guidance on community mitigation measures during pandemic such as social distancing, school closures, and isolation should also need to be developed in collaboration with key stakeholders and technical experts. Besides we also need to focus on our effort to continue to strengthen leadership capacity for pandemic influenza response by regularly outlining the scope of authority and key responsibilities by holding table top and other 
exercises at different levels, involving technical experts and stakeholders and issuing and updating national guidelines for planning based on the latest science and ethical guidelines. As proposed in the national plan public health laws also need to be reviewed and made necessary revision to accommodate the framework and authorities through which the government will take measures to contain the pandemic. The Pandemic Preparedness Plan demarcated several essential activities as well as significant amount of new funding that will result in improved readiness. However, preparedness in all level and response targets cannot be achieved without increased, long-term sustainable funding. Many of the activities being requested of district and Upazila health authorities and hospitals and other national and local institutions require the certainty that ongoing funding is assured. Continuing investments in activities leading to national preparedness will ultimately have collateral benefits in defending against other emerging and re-emerging diseases that are perpetual threat to our country.

\section{Prof. Mahmudur Rahman}

Director

Institute of Epidemiology Disease

Control \& Research

Mohakhali, Dhaka

(J Bangladesh Coll Phys Surg 2007; 25 : 53-55) 